\title{
Direct Numerical Simulation of Capillary Rise in Microtubes with Different Cross-Sections
}

\author{
Dongdong Wang, Penguie liu, Jinxin Wang, Xianguun Bao and Huaqiang Chu* \\ School of Energy and Environment, Anhui University of Technology, Ma'anshan 243002, China
}

(Received November 18, 2018; in final form February 26, 2019)

\begin{abstract}
The interface migration of capillary rise in porous media is a complicated problem. The Lucas-Washburn equation provides the relation between the height and the time in early stage with small height, and some restrictions are introduced in this equation. However, with the development of experiments and simulations, the impact of shapes and dynamic contact angle on capillary rise can be carried out from microscopic view. In this paper, a multiphase lattice Boltzmann model is applied to simulate the capillary rise in microtubes with different crosssections. Through the simulations of three-dimensional capillary rise, the effect of the cross-section shapes on the equilibrium height is compared, and the contact angle hysteresis is found in the angular capillary tubes, that the contact angle of the main terminal meniscus in equilibrium height is larger than the static contact angle.
\end{abstract}

DOI: 10.12693/APhysPolA.135.532

PACS/topics: capillary rise, different cross-sections, deformed interface, contact angle hysteresis

\section{Introduction}

Porous media are widely used in various modern technologies. The capillary suction performance of porous media is a subject of common interest. The interface migration of capillary rise in porous media is a complicated problem, and many related study have been carried out [1-4]. The capillary suction optimization is the improvement of the two-phase interface migration in microchannels, and the basis is the optimization for capillary rise in microtube. Some new models have been presented, such as a multiscale diffuse-interface model for capillarity-driven flow in porous media presented by Roudbari et al. [5]. Their model is based on averaging of the equations of motion for binary fluid with a diffuse interface according to the Navier-Stokes-Cahn-Hilliard equations, but a precise description of microscale flow is not the interest of their model.

For a detailed understanding, capillary rise is the key to improve the capillary suction performance of porous media. The classic description of capillary rise is given by the Lucas-Washburn equation [6], assuming that the contact angle preserves the equilibrium value at all times. The Lucas-Washburn equation provides the relation between the height and time in early stage with small height, corresponding to squared height in proportion to the time. The capillary rise process is activated by capillary force, which is balanced by viscous force, inertial force, and gravity. With major forces considered only, the analytic solutions can be obtained for four time stages, including purely inertial time stage, viscous-inertial time stage, purely viscous time stage, and viscous-gravitational time stage.

*corresponding author; e-mail: hqchust@163.com
The influence of the inertia and the gravity is neglected in the Lucas-Washburn equation. However, the inertia force needs to be considered in the very beginning of capillary rise. The foregoing ground experiments are mainly carried out using small diameter tubes under normal gravity, and then the $h^{2} \sim t$ behavior predicted by the Lucas-Washburn equation is observed mainly. Fortunately, the microgravity experiments can provide the very initial behavior. Before the $h^{2} \sim t$ behavior in the initial stage of capillary rise, the relation of two different stages are found in microgravity experiments that is height in proportion to squared time and time, respectively [7].

In the initial stage of capillary rise, the inertia and viscous force are in a continuous change, and the meniscus is unstable. The contact angle needs a process to reach the equilibrium contact angle, and the dynamic contact angle has been studied in capillary rise [8-12]. In order to measure contact angle, capillary rise experiments have been carried out in single capillaries and in columns of packed powder [8]. During capillary rise, the contact angle is always larger than the static contact angle, and large values may be up to $60^{\circ}$, although their expected value may be $0^{\circ}$. The dynamic contact angle can be expressed by the function of $\mathrm{Ca}$ number, and some assumptions are proposed based on this relation [9]. The experiments of direct measurement of dynamic contact angle and its variation with the velocity of the moving meniscus can be found from Heshmati's work [10]. Through analyzing the effects of dynamic contact angle on liquid infiltration into horizontal capillary tubes, a semi-analytical solution is proposed by Hilpert [11]. Based on velocity-dependent dynamic contact angle relations, Popescu et al. [12] proposed four expressions to explain the lack of consistent experimental evidence for deviations in the rate of capillary rise from the Washburn equation. Since, the exact form of the dependence is still a question of debate, especially for irregular shapes, the model requires further investigation. 
The Lucas-Washburn equation is obtained with circular cross-section capillaries. However, many other factors contribute to the complexity of the capillary rise process, such as dynamic contact angle, and irregular crosssections. As a result, continuous interest maintains on the topic, though the capillary rise has been studied for many years. Since the Lucas-Washburn equation cannot be applied at the two ends of the liquid column in the capillarity, Levine et al. [13] have presented a general Washburn formula for the rate of rise of the meniscus as a function of height in a cylindrical tube. The meniscus exerts an extra drag increasing the effective height. In the late stage of capillary rise, the gravity need to be considered, and an analytic solution of capillary rise restrained by gravity is proposed by Fries et al. [14]. By introducing tortuosity and fractal dimension, capillary rise in a single tortuous capillary has been studied [15]. Their results show that the accumulated weight of liquid imbibed into a single tortuous capillary is independent of the shape of a capillary in the early rising stage.

The meniscus of a certain liquid behaves differently in various cross-section capillary tubes. An empirical solution of the surface tension from capillary rise in rectangular tubes is obtained by experiments [16]. In noncircular capillary tubes, the arc menisci in the corners and the main terminal meniscus have different behaviors, and rapid advancement of arc menisci ahead of main terminal meniscus impacts the dynamics of rise. Capillary rise in different sinusoidal capillaries is simulated by Amara et al., and the results show that the meniscus reaches different equilibrium positions [17]. Figliuzzi et al. [18] present a model to describe the capillary rise of a liquid in a capillarity of varying circular cross-section, and used the optimal control theory to design the optimized capillaries. Berthier et al. [19] derive a general condition for the establishment of spontaneous capillary rise in any composite microchannel of constant cross-section, including the microchannel comprising different wall materials and even open parts. For a given inlet radius, the composite shape can realize the minimum imbibition time. Gorce et al. [20] find that imbibition into tubes with a power-law relationship between the radius and axial position generally occurs more quickly than a constantradius tube. Berthier et al. [21] propose a general expression for the determination of the velocities of spontaneous capillary flows in composite and confined microchannels of arbitrary shapes. But this expression is restricted in sharp corners, due to the entrainment effect on the bulk of the flow.

The initial stage of capillary rise, dynamic contact angle and the shape of capillary tubes have important impact on the capillary rise. The lattice Boltzmann method has microscopic and mesoscopic features that facilitate the processing of irregular boundary conditions, which can be applied to study this problem from microscopic view. Santos et al. [22] present several simulations results of capillary invasions in two- and three dimensions by using lattice Boltzmann method. The velocity field near the solid, the dynamic contact angle, and the boundary conditions are investigated, and their work indicated the potential of lattice Boltzmann method in capillary invasion dynamic studies. Wolf et al. [23] simulate the capillary rise between parallel plates using lattice Boltzmann method. Some discrepancies in the first stages of capillary rise are found when the distance between parallel plates is large. Their results indicate that the inclusion of long-range forces does not change significantly the fluid flow dynamics at the mesoscopic level. Lu et al. [24] develop a multiphase lattice Boltzmann method to investigate capillary rise dynamics, and the numerical results show quantitative agreement with experimental data. The effects of gravity, adhesion, surface tension, viscous drag, inertia, and the tube radius on the initial differences are analyzed in detail to identify the mechanisms in their work.

For the study of capillary rise, it can be seen from the above that the relation between liquid height and time is obtained mainly. The dynamic contact angle can be obtained in circular tube, but rarely is analyzed in angular tubes.

When the capillary rise process is completed, the liquid weight inside the microtube is the balance between the capillary force and the gravity, and the force equilibrium equation can be expressed as

$$
P \sigma \cos \theta=\Delta \rho A h g,
$$

where $A$ is the cross-sectional area, and $P$ is the wetted perimeter of the cross-section.

Introducing the hydraulic diameter $D_{H}=\frac{4 A}{P}$, and then the equilibrium height of capillary rise $h=\frac{4 \sigma \cos \theta}{\Delta \rho g D_{H}}$.

In this simulation, the microtubes are arranged in different geometrical shapes with the same hydraulic diameter. If the meniscus follows the same pattern of distribution, the capillaries can reach the same equilibrium height. But according to the previous research, the corner of capillaries has better wetting ability and different heights will be achieved. The multiphase lattice Boltzmann model is applied and the equilibrium liquid height will be compared in the threedimension microtubes in this paper. The dynamic contact angle and the impact of shapes of microtubes are also studied.

\section{Multiphase lattice Boltzmann model}

\subsection{Multiphase model}

The Shan-Chen multiphase model is used in this paper. With the Bhatnagar-Gross-Krook (BGK) approximation, the lattice Boltzmann equation can be written as

$$
\begin{aligned}
& f_{i}\left(\boldsymbol{x}+\boldsymbol{e}_{i} \Delta t, t+\Delta t\right)= \\
& \quad f_{i}(\boldsymbol{x}, t)-\frac{1}{\tau}\left[f_{i}(\boldsymbol{x}, t)-f_{i}^{e q}(\boldsymbol{x}, t)\right],
\end{aligned}
$$


where $f_{i}(\boldsymbol{x}, t)$ is the density distribution function related to the discrete velocity direction $i$, and $\tau$ is a relaxation time, which is related to the kinematic viscosity by $v=$ $c_{s}^{2}(\tau-0.5) \Delta t$.

The equilibrium distribution function is calculated as

$$
f_{i}^{e q}(\boldsymbol{x}, t)=\omega_{i} \rho\left[1+\frac{\boldsymbol{e}_{i} \cdot \boldsymbol{u}}{c_{s}^{2}}+\frac{\left(\boldsymbol{e}_{i} \cdot \boldsymbol{u}\right)^{2}}{2 c_{s}^{4}}-\frac{(\boldsymbol{u})^{2}}{2 c_{s}^{2}}\right] .
$$

The macroscopic parameters can be calculated as

$$
\begin{aligned}
& \rho=\sum_{i} f_{i}, \\
& u=\frac{1}{\rho} \sum_{i} f_{i} e_{i} .
\end{aligned}
$$

The equilibrium velocity of the fluid is calculated from

$$
u^{e q}=u+\frac{F_{\alpha} \tau}{\rho}
$$

and the actual fluid velocity is calculated by

$$
u^{*}=u+\frac{F_{\alpha} \Delta t}{2 \rho} \text {. }
$$

In Eq. (7), the force $F_{\alpha}$ includes the inter-particle force and the external force.

A D3Q19 model is applied in this study, and the discrete velocity is shown in Fig. 1.

The Caenahan-Starling (C-S) equation of state (EOS) is used for the multiphase fluid, which is defined as:

$$
p=\rho R T \frac{1+b \rho / 4+(b \rho / 4)^{2}-(b \rho / 4)^{3}}{(1-b \rho / 4)^{3}}-a \rho^{2},
$$

with $a=0.4963 R^{2} T_{c}^{2} / p_{c}$, and $b=0.18727 R T_{c} / p_{c}$.

The inter-particle force is defined as:

$$
F(x, t)=-G \psi(x, t) \sum_{i} w_{i} \psi\left(x+e_{i} \Delta t, t\right) e_{i},
$$

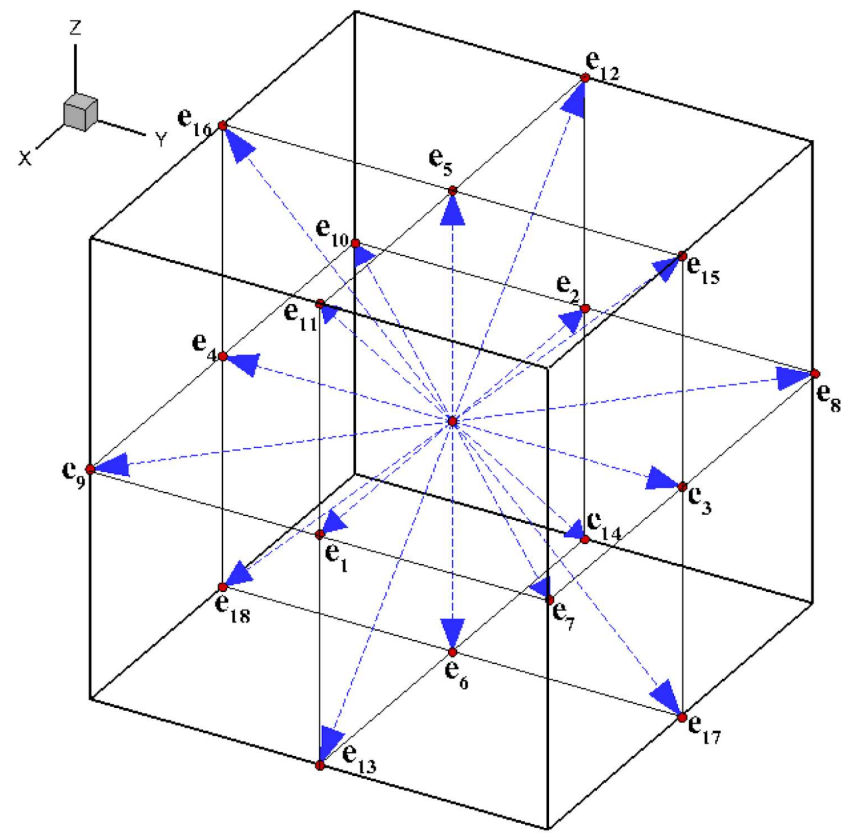

Fig. 1. Discrete velocity model in D3Q19. where $G$ is a parameter that controls the strength of the inter-particle force and $\psi$ is a mean-field potential expressed as

$$
\psi(\rho)=\sqrt{\frac{2\left(p-\rho c_{s}^{2}\right)}{G c_{s}^{2}}} .
$$

\subsection{Surface tension}

The equilibrium states of four droplets with different initial diameters are simulated in a three-dimensional space, then the stable internal and external pressure difference and radius of the droplets are obtained numerically. According to the Young-Laplace equation $\Delta p=2 \sigma / R$, the results of the calculated surface tension are shown in Fig. 2. The average surface tension is $0.00463 \mathrm{mu} / \mathrm{ts}^{2}$ (mu - mass unit, ts - time step), and the maximum relative error is about $2 \%$. The calculated surface tension will be used to ensure the same Ca (capillary) number and Bo (bond) number in the simulation and the real system.

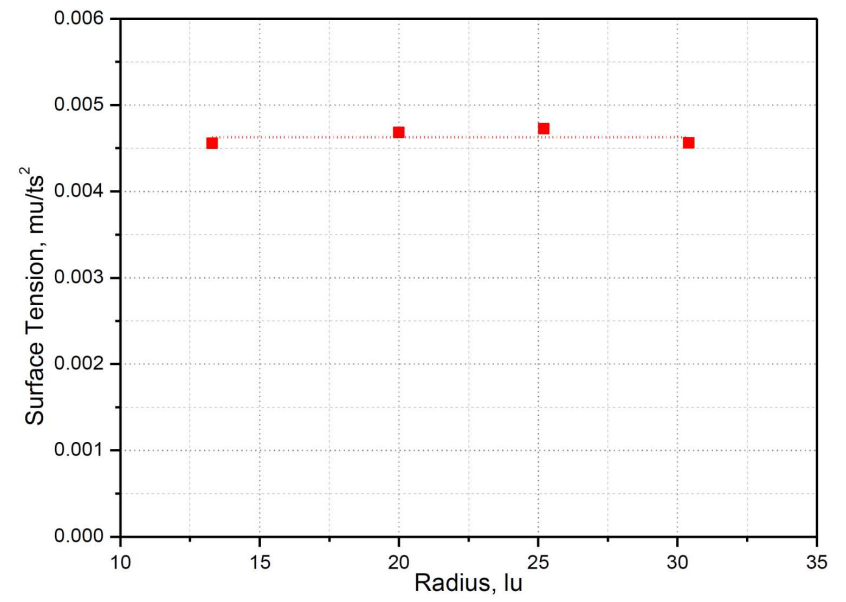

Fig. 2. Surface tension calculation from 3D droplet simulations.

\subsection{Contact angle}

When a droplet comes into contact with a solid wall, the equilibrium contact angle will form in the three-phase contact line. In this Shan-Chen multiphase model, a various "wall density" $\rho_{w}$ is chosen to obtain the desired contact angle. For solid nodes, the bounce-back boundary condition is used. Since the liquid-vapor interface owns certain thickness, the interface is defined at which the fluid density is $50 \%$ of the liquid density [24]. Furthermore, the pre-wetting film forming on the solid wall, the large error happens in the calculation of contact angle as shown in Fig. 3.

\section{Results and discussions}

Three microtubes with the circular, rectangular, and triangular cross-section are studied, respectively, and the geometry can be seen in Fig. 4. The three different crosssections are shown clearly in Fig. 5. Each node can be 


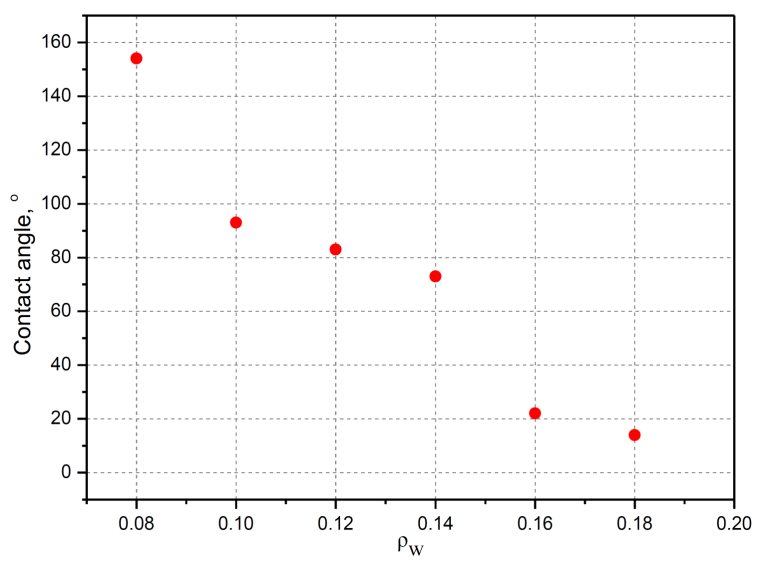

Fig. 3. Relation between contact angle and wall density.


Fig. 4. Three microtubes with different cross-sections.



Fig. 5. Cross-sections in the three simulated microtubes. occupied only by the fluid or the solid. The black nodes represent the solid wall, and the white is the flowing space in Fig. 5. In this setting, the rectangular microtube owns the desired hydraulic diameter, but the circular and triangular microtubes have about $8 \%$ lower than the desired value in diameter. With the same hydraulic diameter, the circular microtube owns the smallest area and perimeter, and the triangular owns the biggest ones. In the simulating zone, the upper and lower boundaries are wall. The periodic boundary condition is applied to the other boundaries, and the bounce-back boundary condition is applied to the fluid-solid interface

\subsection{Equilibrium height}

The simulating equilibrium heights in the three microtubes are shown in Fig. 6. The red stands for the wall, the blue for the non-wetting fluid, and the purple for the wetting fluid. It can be seen that the equilibrium height in the circular microtube is the largest, and the lowest height is in the triangular microtube. The corners in the rectangular and triangular microtubes show better capillary pumping performance, especially in triangular one. The reason is that the meniscus rises in corners following a universal $h^{3} \sim t$ law without saturation [25]. The asymmetry of the meniscus in the triangular microtube appears obviously.

The relation of the hydraulic diameter for cylinder is $\frac{P}{A}=\frac{2}{R}$. According to Eq. (1), the equilibrium height of capillary rise in the cylinder is the Jurin height, which is expressed as $h=\frac{2 \sigma \cos \theta}{\Delta \rho g R}$.

For the circular microtube, the theoretical equilibrium height is $55.4 \mathrm{lu}$ for the desired circular, and the simulated result is $58.9 \mathrm{lu}$, which is about $6 \%$ higher than the theoretical value. For the simulated geometry, the circular structure is composed of nodes, and the theoretical equilibrium height for the actual geometry is $58.0 \mathrm{lu}$ (lu - lattice unit), which is very close to the simulated result. For the rectangular and triangular microtube, the equilibrium height cannot be predicted by the above equation. The wetted perimeter of the cross-section becomes irregular and is difficult to be expressed in the corners, as shown in Fig. 6.

The dimensionless parameters are chosen as $h^{*}=\frac{h}{h_{\max }}$, and $t^{*}=\frac{\rho g R^{2} t}{8 \mu h_{\max }}$.

When the whole capillary rise process is considered, the gravity cannot be ignored and the Lucas-Washburn equation is inapplicable. As the comparison among the Fries model, experimental results and experimental data (Fig. 7), for $t^{*}$ between 0.2 and 1.4 some deviation to lower results can be seen. Their explanation is a higher friction than expected originating from surface roughness, the neglect of entry effects or assuming a constant contact angle [14]. In the experiments of capillary rise, the tubes, open on bottom and top, are immersed into the liquid from above. Then the surface oscillation will happen at the beginning of capillary rise [26]. However, in the simulation, an initial horizontal interface is set 

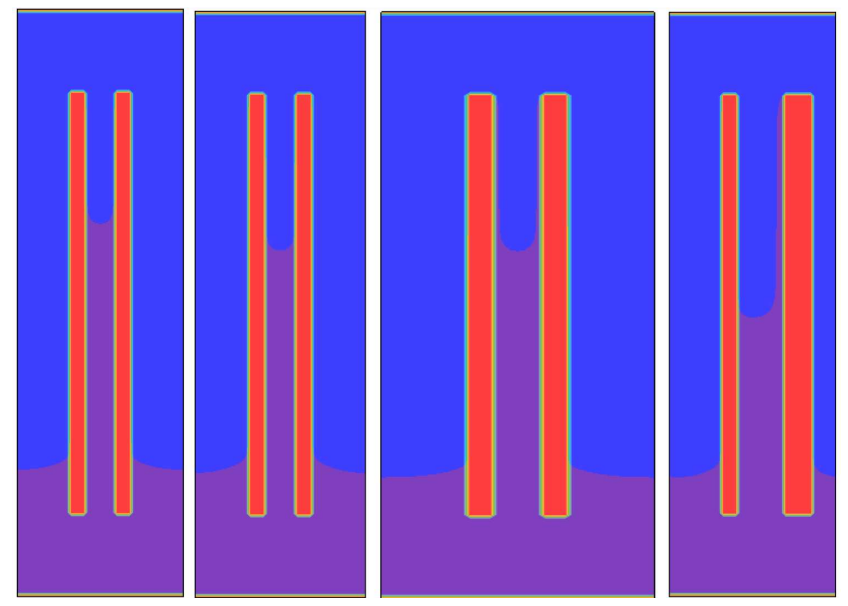

Fig. 6. Equilibrium height in the three microtubes: (a) circular microtube, (b) rectangular microtube, (c) diagonal line in rectangular microtube, (d) triangular microtube.

inside and outside of microtubes. These factors are different among model, experiment, and simulation, which may cause some deviation in results.

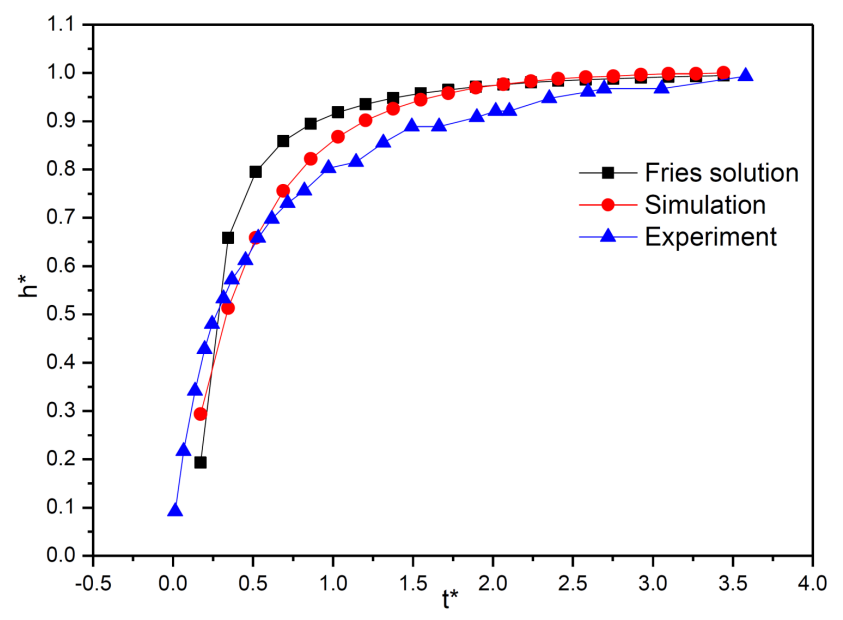

Fig. 7. Comparison of theoretical, experimental [14], and simulated results of capillary rise.

It can be seen from Fig. 8 that, for different tubes with the same hydraulic diameter, the equilibrium height of circular tube is largest and the triangular one is lowest. In the simulation, the equilibrium height for circular, rectangular and triangular cross-section is 58.9, 52.9, and $36.9 \mathrm{lu}$, respectively. For the three microtubes with different cross-sections, the circular cross-section owns the smallest wetted area and perimeter, which corresponds to the largest viscous force and smallest capillary force. But, the results show that the circular cross-section reaches the largest equilibrium height. For the circular and rectangular microtubes, the distance between the center and the wall is the same, but the corner in the rectangular one has larger distance and smaller dynamic contact angle.

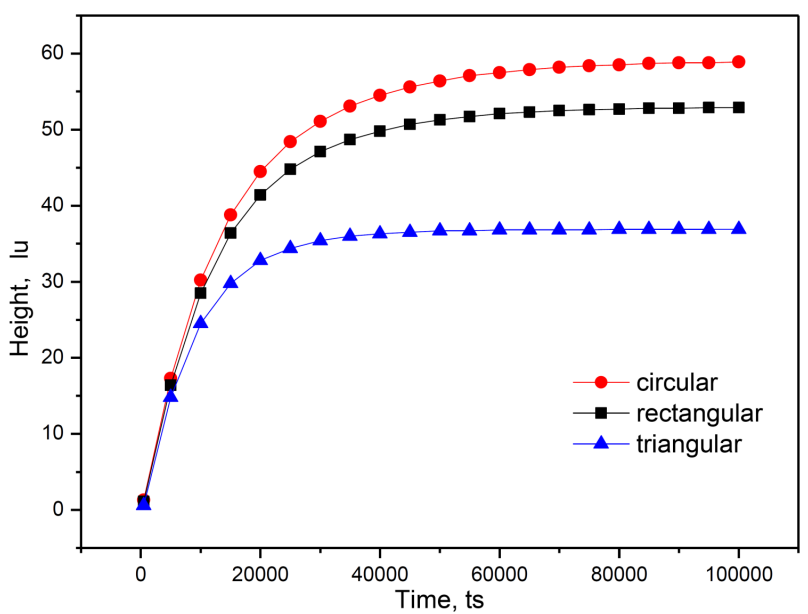

Fig. 8. Capillary rise height of the central meniscus as a function of time.

According to Princen's model [27], the rising liquid includes the liquid column up to the central meniscus and the fingers along the corners, and the equilibrium height for the central meniscus is

$$
h=(2+\sqrt{\pi}) \frac{\kappa^{-2}}{a},
$$

where $a$ is side length, and $\kappa^{-1}=\sqrt{\sigma / \rho g}$. For rectangular cross-section, the theoretical rise height is $51.8 \mathrm{lu}$, which is about $2 \%$ lower than the simulated value (52.9 lu).

The mean height is defined by the suction volume in the three cross-sections. When the mean height is applied, the equilibrium height in the circular, rectangular and triangular microtube is $65.0,57.7$, and $46.0 \mathrm{lu}$, respectively. Compared with the equilibrium height of the central meniscus, the mean equilibrium height has the relative increase of about $10.4 \%, 9.1 \%$, and $24.7 \%$, respectively. The results are directly related with the corners in the three microtubes.

The mean rising heights of the central meniscus in three microtubes increasing from 500 ts to 100000 ts are shown in Fig. 9. It can be seen from Fig. 9 that there are some differences in the initial stage, where a bigger height appears in the triangular microtube. This is because of that the gravity can be ignored, and the triangular microtube owns the largest wetted perimeter of the three microtubes, corresponding to the largest capillary force.

The Lucas-Washburn equation shows the relations in the initial stage of capillary rise. According to Washburn's equation, $h^{2}$ must vary linearly with time [8]. But in the very initial stage of capillary rise, the process is governed by inertial force, leading to a linear variation of the height versus time. The relations of $h \sim t^{2}$ and $h \sim t$ are found in many experiments, which also can be seen from Fig. 10. But there is an initial stage of interface falling, corresponding to the central meniscus lower than the initial position. 


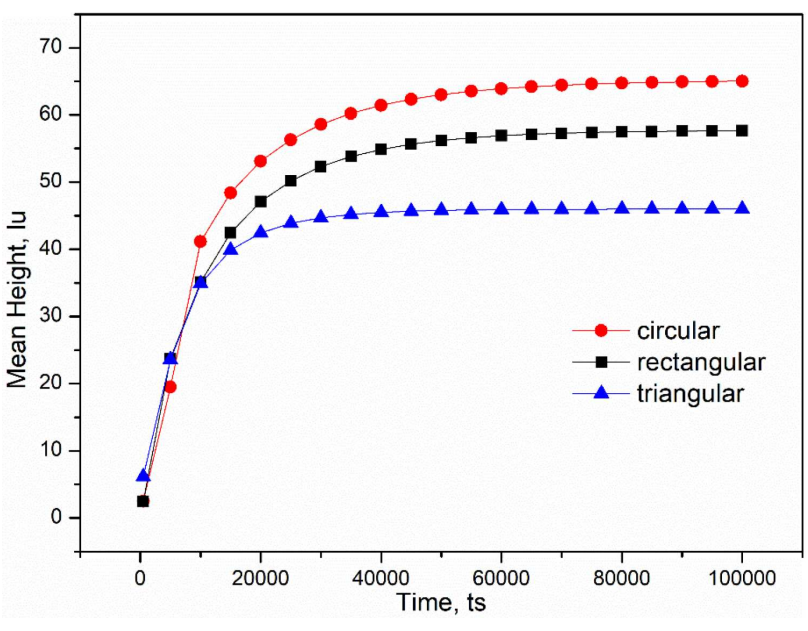

Fig. 9. Mean rising height of the central meniscus as a function of time.

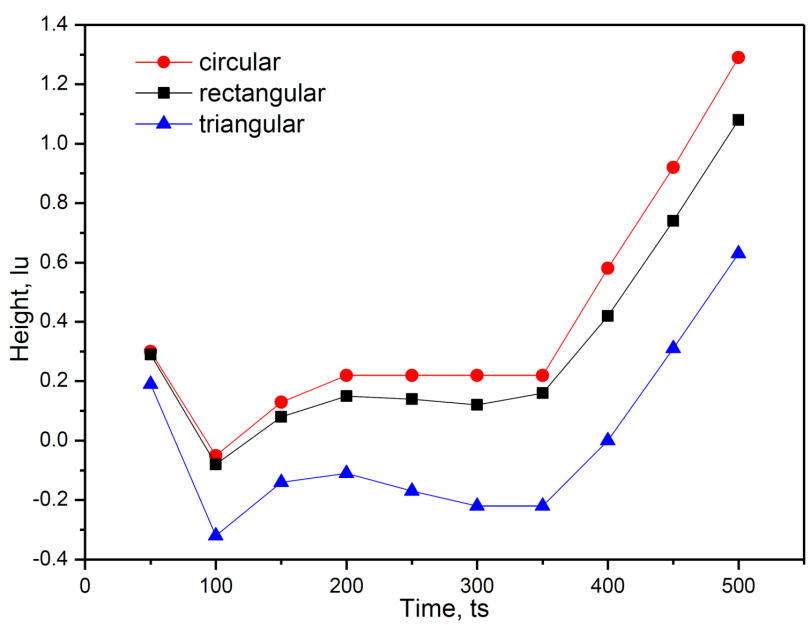

Fig. 10. Rising height varies with time in the initial stage of capillary rise.

\subsection{Dynamic contact angle}

The contact angle of the main terminal meniscus is defined as the dynamic contact angle in this paper, which is the largest contact angle vertically in the microtube during capillary rising. In the rectangular and triangular tubes, the smallest contact angles are in the corners. It can be seen from Fig. 11 that the dynamic contact angle decreases linearly to a relative stable value in the capillary rise process. But for the three different microtubes, the equilibrium contact angles have great differences, although the expected contact is the same. The maximum contact angle in the equilibrium height is in the triangular microtube. The equilibrium contact angle in circular microtube is very close to, but slightly larger than the static contact angle. In the initial stage, the largest contact angle appears in the triangular microtube, which is consistent with the lowest height of the central meniscus in the same condition.

In Xue's experiments, the dynamic contact angle decreased and then reached a plateau value over time in the

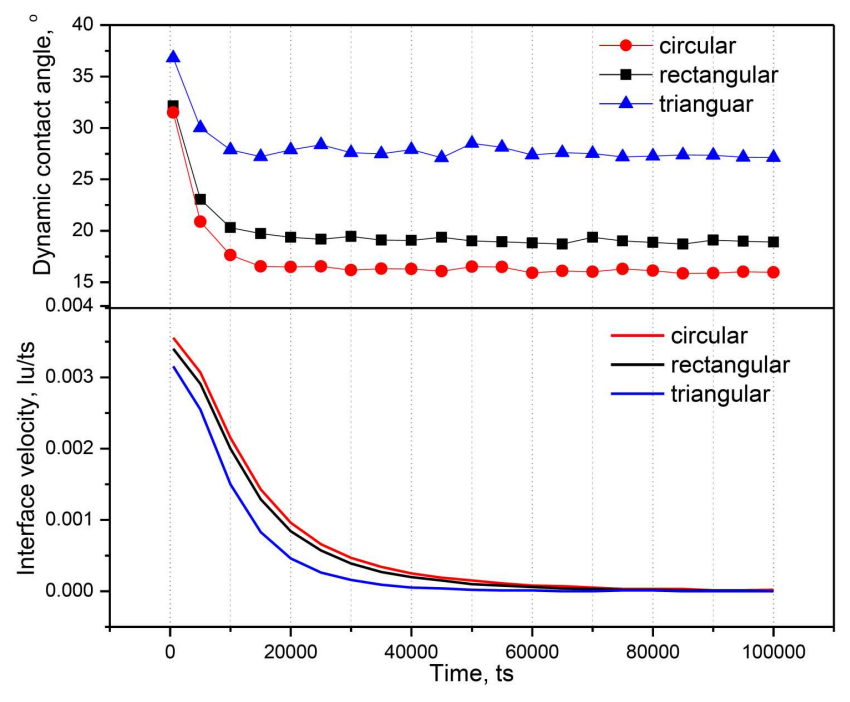

Fig. 11. Dynamic contact angle and interface velocity varies with time.

initial stage of capillary rise, which had a very surprising difference between the experimental results and the expected results [28]. In their work, the contact angle is deduced from the experimental data in the capillary tube with the radius of $0.15 \mathrm{~mm}$. Their proposed reasons are the contact angle hysteresis and the dynamic contact angle effects.

The contact angle hysteresis can be found in the angular capillary tube, in which the stable contact angle is larger than the equilibrium contact angle. The phenomenon cannot be found in the two-dimensional simulation, and is not easy to be found in the experiments due to the small differences between the equilibrium contact angles and the static contact angle. In the threedimensional capillary rise, the force balance of the contact line is complicated. Considering the corner, the curvatures of the arc meniscus and the main terminal meniscus are asymmetry, and then the contact angle hysteresis may happen along rising direction.

\subsection{Interface rising velocity}

In the capillary rise of the three microtubes, the circular capillary takes the largest equilibrium height and the longest time to the equilibrium position, and it owns the largest interface rising velocity at the same time (Fig. 11).

For the interface migration in circular tubes, the dynamic contact angle is related to the static contact angle and the capillary number $\mathrm{Ca}$, which can be expressed as

$$
\cos \theta_{d}=\cos \theta_{0}-\alpha \mathrm{Ca}^{\beta},
$$

and the capillary number $\mathrm{Ca}=\frac{\mu}{\sigma} \frac{\mathrm{d} h}{\mathrm{~d} t}$, where $u=\frac{\mathrm{d} h}{\mathrm{~d} t}$ is the interface moving velocity, $\theta_{0}$ and $\theta_{d}$ is the static and dynamic contact angle, respectively.

The dynamic contact angle is affected by the velocity and the shape. In this simulation, the parameters in the equation of the dynamic contact angle and $\mathrm{Ca}$ are obtained by fitting the curves, and $\alpha=3.51267 \times 10^{-5}$, 
$\beta=-0.29834$. But in the previous study $[9,23]$, the parameter $\beta$ is usually equal to some positive value, which is very different from the present results. One of the proposed reasons is the contact angle hysteresis. The defined dynamic contact angle is the advancing contact angle for capillary rise, but is the receding contact angle for the wetting of the corners. Another is the interface deforming effects. The previous relation is from the experimental results for the dynamic contact angle in the symmetric interface. The dynamic contact angle defined in this paper is affected by the arc meniscus, and the main meniscus is a deforming interface, which causes the differences between the equilibrium contact angles and the static contact angle during dynamic interface migration.

\section{Conclusions}

Three microtubes with circular, rectangular, and triangular cross-section are set with the same hydraulic diameter, and then capillary rise in these microtubes are simulated using multiphase lattice Boltzmann model in three dimensions. The results are analyzed and compared with previous work. Some conclusions can be given as follows:

1. For the microtubes with the same hydraulic diameter, the equilibrium height of capillary rise is largest in the circular microtubes.

2. In the very beginning of capillary rise, the interface inside the microtubes may lower than the initial height, and the capillary rising column is related to the shape of the cross-section.

3. The contact angle hysteresis has been found in angular microtubes, that the contact angle of the main terminal meniscus is larger than the static contact angle.

Further work must be done to understand the contact angle hysteresis in the condition of deformed interface.

\section{Acknowledgments}

The authors greatly appreciate the financial support provided by National Natural Science Foundation of China (No. 51706001) and Provincial Natural Science Foundation of Anhui (KJ2016A095).

\section{References}

[1] V. Ganesan, H. Brenner, Phys. Rev. Lett. 81, 578 (1998).

[2] G. Martic, J.D. Coninck, T.D. Blake, J. Coll. Interface Sci. 263, 213 (2003).

[3] T. Delker, D.B. Pengra, W. Pz, Phys. Rev. Lett. 76 2902 (1996).
[4] M. Panfilov, I. Panfilova, Trans. Porous Media 58, 87 (2005).

[5] M. Shokrpour Roudbari, E.H.V. Brummelen, C.V. Verhoosel, Comput. Fluids 141, 212 (2016).

[6] E.W. Washburn, Phys. Rev. 17, 273 (1921).

[7] M. Dreyer, A. Delgado, H.J. Path, J. Coll. Interface Sci. 163, 158 (1994).

[8] A. Siebold, M. Nardin, J. Schultz, A. Walliser, M. Oppliger, Coll. Surf. 161, 81 (2000).

[9] S.V. Mourik, A.E.P. Veldman, M.E. Dreyer, Microgravity 17, 87 (2005).

[10] M. Heshmati, M. Piri, Langmuir 30, 14151 (2014).

[11] M. Hilpert, J. Coll. Interface Sci. 337, 131 (2009).

[12] M.N. Popescu, J. Ralston, R. Sedev, Langmuir 24, 12710 (2008).

[13] S. Levine, J. Lowndes, E.J. Watson, G. Neale, J. Coll. Interface Sci. 73, 136 (1980).

[14] N. Fries, M. Dreyer, J. Coll. Interface Sci. 320, 259 (2008).

[15] C. Jian-Chao, Y. Bo-Ming, M. Mao-Fei, L. Liang, Chin. Phys. Lett. 27, 054701 (2010).

[16] K.S. Birdi, D.T. Vu, A. Winter, A. Nørregård, Coll. Polym. Sci. 266, 470 (1988).

[17] M.E.A. Ben Amara, P. Perre, S.B. Nasrallah, J. Porous Media 19, 453 (2016).

[18] B. Figliuzzi, C.R. Buie, J. Fluid Mech. 731, 142 (2013).

[19] J. Berthier, K.A. Brakke, E. Berthier, Microfluid. Nanofluid. 16, 779 (2013).

[20] J.B. Gorce, I.J. Hewitt, D. Vella, Langmuir 32, 1560 (2016).

[21] J. Berthier, D. Gosselin, E. Berthier, Microfluid. Nanofluid. 19, 497 (2015).

[22] L. Santos, F.G. Wolf, P.C. Philippi, J. Statist. Phys. 121, 197 (2005)

[23] F.G. Wolf, L. Santos, P.C. Philippi, J. Coll. Interface Sci. 344, 171 (2010).

[24] G. Lu, X.-D. Wang, Y.-Y. Duan, Coll. Surf. A 433, 95 (2013)

[25] A. Ponomarenko, D. Quéré, C. Clanet, J. Fluid Mech. 666, 146 (2011)

[26] M. Stange, M.E. Dreyer, H.J. Rath, Phys. Fluids 15, 2587 (2003).

[27] J. Bico, D. Quere, J. Coll. Interface Sci. 247, 162 (2002).

[28] H.T. Xue, Z.N. Fang, Y. Yang, J.P. Huang, L.W. Zhou, Chem. Phys. Lett. 432, 326 (2006). 